\title{
BVS5000 support after cardiac transplantation
}

\author{
Jason A. Petrofski, MDa \\ Vijay S. Patel, MD ${ }^{\mathrm{a}}$ \\ Stuart D. Russell, $M D^{b}$ \\ Carmelo A. Milano, MD ${ }^{\mathrm{a}}$
}

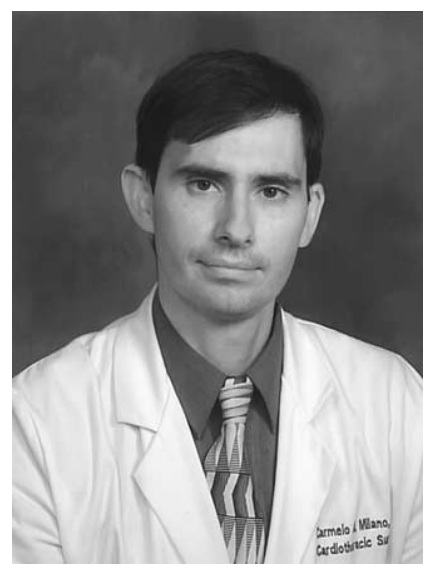

Dr Milano
From the Division of Cardiothoracic Surgery, Department of Surgery, ${ }^{\mathrm{a}}$ and Division of Cardiology, Department of Medicine, Duke University Medical Center, Durham, NC.

Received for publication May 22, 2002; revisions requested July 30, 2002; revisions received Aug 16, 2002; accepted for publication Sept 23, 2002.

Address for reprints: Carmelo A. Milano, MD, Assistant Professor of Surgery, Department of Surgery, The Duke University Medical Center, Box 3043, Durham, NC 27703 (E-mail: milan002@mc.duke.edu).

J Thorac Cardiovasc Surg 2003;126:442-7

Copyright $\odot 2003$ by The American Association for Thoracic Surgery

$0022-5223 / 2003 \$ 30.00+0$

doi:10.1016/S0022-5223(02)73613-1
Objective: This study examines short-term mechanical assist device support for cardiac transplant patients and compares their outcomes with nontransplant patients requiring similar support.

Methods: Of 350 cardiac transplant patients at our institution, 7 patients required mechanical ventricular assistance with the Abiomed BVS5000 assist device (Abiomed, Inc, Danvers, Mass) after transplant secondary to severe acute rejection with cardiogenic shock $(n=4)$ or primary graft failure $(n=3)$. Recovery of ventricular function, survival to discharge, and complications were determined for the transplant group and compared with a second group comprising all other patients supported with the BVS5000 at our institution $(\mathrm{n}=15)$. Additionally, the results of prior series reporting mechanical ventricular support of the failing transplant heart are reviewed.

Results: Demographics and duration of support were similar between the groups. The transplant group had a higher wean rate from device relative to the nontransplant group $(100 \%$ versus $13 \%$; $P<0.01)$. Five of 7 in the transplant group achieved survival to discharge (71\%), relative to 5 of 15 in the nontransplant group (33\%). Complications between the two groups were similar, although the transplant group experienced a higher rate of renal insufficiency (57\% versus $13 \%, P=0.05$ ).

Conclusion: Severe acute rejection with cardiogenic shock and primary graft failure are two conditions that may warrant mechanical ventricular support in the cardiac transplant patient. Transplant patients with these conditions have a high rate of ventricular functional recovery, greater than nontransplant patients supported with the same device and for a similar period of time. Although the incidence of renal insufficiency was higher, the majority of transplant patients who were supported with the BVS5000 achieved survival to discharge.

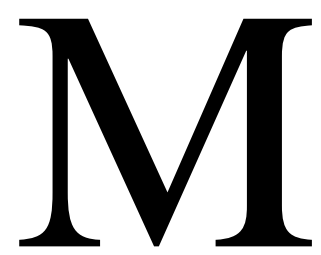

echanical ventricular support is infrequently required for recipients of cardiac transplantation. However, two conditions may necessitate mechanical ventricular support for the transplanted heart: severe acute rejection with cardiogenic shock and primary graft failure. After initial encouraging reports, more recent studies of mechanical ventricular assistance for these conditions have described relatively poor outcomes. ${ }^{1-7} \mathrm{~A}$ variety of devices have been utilized for ventricular support of these patients ranging from extracorporeal membrane oxygenation (ECMO) to more permanent ventricular assist devices such as the Thoratec device (Thoratec Corporation, Pleasanton, Calif). 
TABLE 1. Brief description for each patient in this study

\begin{tabular}{|c|c|c|c|c|}
\hline Age/gender & Indication & Type of support & Weaned & Outcome \\
\hline \multicolumn{5}{|l|}{ Group A } \\
\hline $53 \mathrm{M}$ & Primary graft dysfunction & BiVAD & Yes & Sepsis/death \\
\hline $51 \mathrm{M}$ & Primary graft dysfunction & RVAD & Yes & Survival to discharge \\
\hline $60 \mathrm{M}$ & Primary graft dysfunction & RVAD & Yes & Survival to discharge \\
\hline $67 \mathrm{~F}$ & Severe acute rejection & BiVAD & Yes & Survival to discharge \\
\hline $42 \mathrm{M}$ & Severe acute rejection & BiVAD & Yes & Survival to discharge \\
\hline $45 \mathrm{~F}$ & Severe acute rejection & BiVAD & Yes & Stroke/death \\
\hline $30 \mathrm{~F}$ & Severe acute rejection & BiVAD & Yes & Survival to discharge \\
\hline \multicolumn{5}{|l|}{ Group B } \\
\hline $72 \mathrm{M}$ & Post-cardiac surgery shock & BiVAD & Yes & Survival to discharge \\
\hline $32 \mathrm{~F}$ & Post-cardiac surgery shock & BiVAD & Yes & Mediastinitis/sepsis/death \\
\hline $58 \mathrm{~F}$ & Post-cardiac surgery shock & RVAD & No & Ventricular failure/death \\
\hline $49 \mathrm{~F}$ & Post-cardiac surgery shock & BiVAD & No & Stroke/death \\
\hline $54 \mathrm{M}$ & Post-cardiac surgery shock & LVAD & No & Transplanted/survival to discharge \\
\hline $55 \mathrm{M}$ & Post-cardiac surgery shock & BiVAD & No & Transplanted/sepsis/death \\
\hline $74 \mathrm{~F}$ & Post-cardiac surgery shock & BiVAD & No & Ventricular failure/death \\
\hline $75 \mathrm{M}$ & Post-cardiac surgery shock & RVAD & No & Ventricular failure/death \\
\hline $37 \mathrm{~F}$ & Acute viral myocarditis & BiVAD & No & Transplanted/death \\
\hline $31 \mathrm{~F}$ & Acute viral myocarditis & BiVAD & No & Transplanted/survival to discharge \\
\hline $50 \mathrm{~F}$ & Cardiomyopathy/arrest & BiVAD & No & Transplanted/survival to discharge \\
\hline $52 \mathrm{M}$ & Cardiomyopathy/arrest & BiVAD & No & Transplanted/survival to discharge \\
\hline $47 \mathrm{M}$ & Cardiomyopathy/arrest & BiVAD & No & Transplanted/death \\
\hline $64 \mathrm{M}$ & Cardiomyopathy/arrest & BiVAD & No & Stroke/death \\
\hline $52 \mathrm{M}$ & Cardiomyopathy/arrest & BiVAD & No & Stroke/death \\
\hline
\end{tabular}

Group A patients were supported with the BVS5000 after cardiac transplantation. All of these patients were weaned from the device after left ventricular recovery. Five of the 7 patients were long-term survivors. Group B comprises all other patients supported with the BVS5000; only 2 were weaned from the BVS5000. Only 1 of these 2 patients survived to discharge. Four other patients who were unable to be weaned underwent subsequent heart transplant with long-term survival. RVAD, Right ventricular assist device; $L V A D$, left ventricular assist device; BiVAD, biventricular assist device.

We now have supported 7 cardiac transplant patients with the Abiomed BVS5000 assist device (Abiomed, Inc, Danvers, Mass). We chose this device due to its ease of implantation and maintenance. This review illustrates the implantation technique of the BVS5000 for cardiac transplant patients. Additionally, the rates of ventricular recovery and survival to discharge for these cardiac transplant patients are compared with a group of 15 nontransplant patients who were supported with the BVS5000 for other indications at our institution. Given the concomitant immunosuppression in the transplant patients, we also compared postimplantation complications between the two groups. Finally, we reviewed the literature for other reports of cardiac transplant recipients requiring ventricular assist device (VAD) support.

\section{Methods}

\section{Patient Groups}

We identified all orthotopic cardiac transplant patients at our institution $(n=350)$ and reviewed the medical records of the 7 patients who required posttransplant support with the Abiomed BVS5000 device. These 7 patients composed group A and either suffered primary graft dysfunction at the time of implantation or experienced severe acute rejection, which failed to improve after inotropic support (Table 1).
Results in this cohort were compared with all other patients at our institution who required ventricular support with the BVS5000 $(\mathrm{n}=15)$. These patients, who comprise group $\mathrm{B}$, had low cardiac output syndrome secondary to (1) post-cardiac surgery cardiogenic shock, (2) acute viral myocarditis, or (3) cardiomyopathy (Table 1).

All patients showed evidence of a low cardiac output state (cardiac index $<2.0 \mathrm{~L} / \mathrm{min} / \mathrm{m}^{2}$ ) despite volume optimization and maximal pharmacologic support (dobutamine 25 to $30 \mathrm{mcg} / \mathrm{kg}$ / min, epinephrine 0.8 to $1.2 \mathrm{mcg} / \mathrm{kg} / \mathrm{min}$, milrinone 0.50 to 0.75 $\mathrm{mcg} / \mathrm{kg} / \mathrm{min}$ ). The vast majority of patients were supported with intra-aortic balloon pumps (IABP; 6/7 in the transplant group, $14 / 15$ in the nontransplant group) prior to BVS5000 implantation. Abiomed BVS5000 support was started only after these less invasive treatments failed to achieve viable hemodynamics.

For those transplant patients with biopsy-proven rejection, high-dose methylprednisolone (Solu-Medrol), increased doses of cyclosporine, and antilymphocyte antibodies were utilized. In addition, humoral rejection was treated aggressively with cyclophosphamide (Cytoxan), intravenous immunoglobin (IVIG), and plasmapheresis. In cases of primary graft failure, plasmapheresis was utilized until biopsy and crossmatch results returned negative for rejection and an immunologic process was ruled out as the etiology of ventricular dysfunction.

For the two transplant patients who experienced primary graft failure necessitating right ventricular assist device (RVAD) sup- 


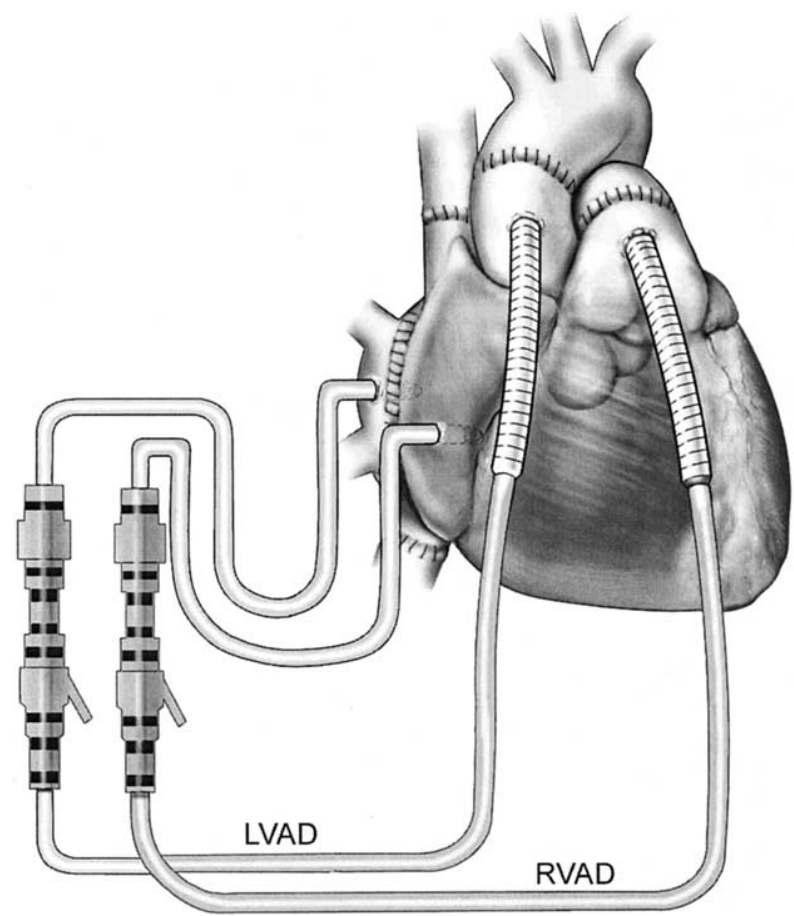

Figure 1. A heart transplant is illustrated and a bicaval implant technique employed. For RVAD support, the body of the right atrium is cannulated away from the superior and inferior vena caval anastomoses. Outflow from the RVAD is to a graft cannula sewn to the main pulmonary artery (PA). The graft is placed just distal to the pulmonary valve but proximal to the anastomosis between the donor and recipient PA. For LVAD support, the recipient left atrial cuff is cannulated adjacent to the right pulmonary vein, providing drainage to the pump. Outflow from the LVAD is to a graft cannula sewn to the recipient aorta just above the aortic valve. RVAD, Right ventricular assist device; $L V A D$, left ventricular assist device.

port, both had less severe left ventricular (LV) dysfunction that ultimately did not require ventricular assist device (VAD) support. In both of these cases inhaled nitric oxide was utilized and failed to achieve viable hemodynamics prior to RVAD placement. Elevated pulmonary vascular resistance was not felt to be the primary etiology for right ventricular (RV) failure.

\section{Definitions}

Weaned is defined as return of native ventricular function enabling discontinuation of the device. Survival to discharge is defined as survival enabling discharge from the hospital but excluding patients who expired after discharge due to complications from the admission during which the device was implanted. (One patient was discharged but within 5 days required readmission secondary to renal failure and subsequently expired during rehospitalization.) Survival 6 months after BVS5000 removal is defined as patients alive 6 months after the device was explanted. No patients were lost to follow-up. Patient age, wean status, duration of support, survival to discharge, survival 6 months after BVS5000 removal, and complications were recorded for each group.
Complications examined for each group included renal insufficiency, pulmonary insufficiency, stroke, bleeding, and sepsis. Renal insufficiency is defined as more than a doubling of serum creatinine during the period of BVS5000 support. Pulmonary insufficiency is defined as the need for mechanical ventilation with a $\mathrm{FiO}_{2} \geq 60 \%$ for more than 24 hours during the period of BVS5000 support. Stroke is defined as any acute focal lesion identified by computed tomography of the brain and/or a new focal deficit identified on physical examination that occurred during the period of BVS5000 support. Postoperative bleeding is reported as the total amount of chest tube output during the first 24 hours after device implantation. Sepsis is defined as positive blood cultures during the period of BVS5000 support or wound infection extending to the sternal closure at any time following BVS5000 implantation.

\section{Surgical Technique}

For all 7 transplant patients (group A), atrial cannulation was employed and outflow graft cannulae were sewn onto the transplanted heart great vessels. The graft cannulae were sewn in an end-to-side manner to the great vessels (aorta and/or pulmonary artery) of the donor heart. The cannulae therefore were placed just distal to the aortic or pulmonic valves and just proximal to the transplant anastomoses (Figure 1). For the aortic anastomosis a side-biting clamp was employed, while vacuum-assisted venous drainage enabled an open anastomosis at the pulmonary artery (the pulmonary artery is sufficiently decompressed that clamping is not required). Others have described placement of the RVAD outflow cannula through the right ventricle and then out through the pulmonary valve. ${ }^{8}$ Our experience with this approach resulted in problematic bleeding around the cannula. Additionally, cannula placement through the right ventricle may be damaging to the right ventricle itself. For these reasons we advocate use of the graft cannula that is sewn easily to the pulmonary artery.

All heart transplants were implanted with a bicaval technique. Therefore, an extensive area of recipient left atrium is present for cannulation adjacent to the right pulmonary veins. We have found left atrial cannulation in this location to be easier in the transplant setting relative to other patients due to the extensive cuff of recipient left atrium. Individual superior and inferior vena caval anastomoses enabled cannulation of the donor heart right atrium without interfering with suture lines (Figure 1).

\section{Literature Review}

A review of the literature was performed by electronic Medline search utilizing the key words heart transplantation and heartassist device, incorporating journal articles published between 1966 and 2002. Case reports of individual patients were not included.

\section{Statistical Analysis}

Student $t$ test was used to compare age, gender, duration of support, and postoperative bleeding between the two groups. Fischer's exact test was used to compare number weaned, survival to discharge, 6-month survival after BVS5000 removal, renal insufficiency, pulmonary insufficiency, stroke, and sepsis between the two groups. A $P$ value $\leq 0.05$ was considered statistically significant. 
TABLE 2. Comparison of demographics/outcomes

\begin{tabular}{lccr}
\hline Demographic/outcome & Group A & Group B & $\boldsymbol{P}$ value \\
\hline Age (in years) & 49.5 & 53.4 & .55 \\
Duration of support (in days) & 5.2 & 5.1 & .86 \\
Number weaned (\%) & $7 / 7(100 \%)$ & $2 / 15(13 \%)$ & $<.01$ \\
Survival to discharge (\%) & $5 / 7(71 \%)$ & $5 / 15(33 \%)$ & .36 \\
Survival 6 months after BVS5000 & $5 / 7(71 \%)$ & $5 / 15(33 \%)$ & .36 \\
$\quad$ removal & & & \\
\hline
\end{tabular}

The number of patients weaned in Group A was significantly higher than Group B $(P<.01)$. Survival to discharge and survival 6 months after device removal, while not statistically significant, both trend toward Group $A$. Additionally, 4 of the 5 patients in Group B who survived did not experience native ventricular recovery, were unable to be weaned from the BVS5000, and ultimately required orthotopic heart transplant. Age and duration of support were not found to be statistically significant between the 2 groups.

\section{Results}

Seven of 350 patients who had undergone cardiac transplant required mechanical ventricular support with the BVS5000. Three of the 7 patients had primary graft failure. In 2 of these 3, graft dysfunction was predominantly right-sided. The remaining 4 patients who received a BVS5000 after cardiac transplantation had severe acute rejection with cardiogenic shock despite conventional treatments (volume loading, inotropic support, etc). Of these 4 patients, two suffered from rejection during the first week after their transplant procedure and a humoral component was identified. The remaining 2 patients experienced an episode of severe rejection more than 1 year after transplant. In these final 2 cases, patient noncompliance with their immunosuppressive regimen was suspected, and the etiology was more typical cellular rejection.

Patients in group A were supported for an average of 5.2 days and all 7 experienced ventricular recovery enabling the device to be weaned (Table 2). One of the 7 patients suffered anoxic brain injury secondary to cardiac arrest that occurred prior to device implantation. This patient died after withdrawal of ventilatory support following graft ventricular recovery. Another patient developed sepsis and profound leukopenia after device removal; multiorgan system failure and death ensued. Concomitant immunosuppression contributed to the complicating leukopenia in this case. The remaining 5 patients each survived to discharge and were alive at follow-up 6 months after device removal.

Group B, the nontransplant group, was comprised of 15 patients with 3 etiologies of ventricular failure: (1) postcardiac surgery shock, (2) acute viral myocarditis, or (3) cardiomyopathy with sudden deterioration and cardiac arrest. In contrast to group $\mathrm{A}$, group $\mathrm{B}$ experienced a much lower rate of weaning from device $(P<0.01)$. Only 2 patients were weaned from the device in group B (13\%). Only 1 of the 15 patients was successfully weaned from the device and survived to discharge $(6 \%)$. Although there were
TABLE 3. Comparison of complications

\begin{tabular}{lccc}
\hline Complication & Group A & Group B & $\boldsymbol{P}$ value \\
\hline $\begin{array}{l}\text { Postoperative bleeding } \\
\quad \text { (mean in mL) }\end{array}$ & 902 & 1076 & .50 \\
Renal insufficiency (\%) & $4 / 7(57 \%)$ & $2 / 15(13 \%)$ & .05 \\
Pulmonary insufficiency (\%) & $1 / 7(14 \%)$ & $2 / 15(13 \%)$ & .95 \\
Stroke (\%) & $1 / 7(14 \%)$ & $3 / 15(20 \%)$ & .29 \\
Sepsis (\%) & $1 / 7(14 \%)$ & $2 / 15(13 \%)$ & .95 \\
\hline
\end{tabular}

4 others who survived to discharge in group B, each of these patients underwent cardiac transplantation after their native ventricle failed to recover. The 5 patients in group B who survived to discharge were alive at follow-up 6 months after device removal.

No statistically significant differences were noted between the 2 groups with regard to age, gender, length of support, survival to discharge, or survival 6 months after BVS5000 removal (Table 2).

Complications for each group included stroke, pulmonary insufficiency, renal insufficiency, sepsis, and postoperative bleeding (Table 3). Complications occurred at similar rates in the two groups, but there was a greater incidence of renal insufficiency in group A $(P=.05)$. None of the patients in either group required long-term renal dialysis. No instances of device failure occurred in either group.

\section{Discussion}

Multiple studies have documented successful use of the Abiomed BVS5000 device for patients who fail to wean from cardiopulmonary bypass following conventional cardiac surgery procedures. ${ }^{9-12}$ Indeed, post-cardiac surgery cardiogenic shock or failure to wean from cardiopulmonary bypass are the most common indications for this device in previous series as well as among patients at our institution. Use of the device in cardiac transplant patients has not been extensively reviewed and presents new challenges. Preservation of renal function may be more difficult given the concomitant use of cyclosporine or tacrolimus. Furthermore, infectious complications that represent a major problem for VADs in general could pose even greater threats in immunosuppressed cardiac transplant patients. Finally, the capability of the transplanted heart to recover from severe acute rejection with cardiogenic shock or after primary failure of the graft has not been extensively studied. Therefore, this report reviews our results when utilizing the BVS5000 to support cardiac transplant patients.

Potential indications for BVS5000 ventricular support in the cardiac transplant population are primary graft failure and severe acute rejection with cardiogenic shock. Fortunately, these conditions are uncommon. ${ }^{13}$ For primary graft failure, early utilization of percutaneous IABP and inhaled nitric oxide are advocated. These are important noninvasive 
TABLE 4. Single-institution studies reporting the outcomes of VAD support after cardiac transplant

\begin{tabular}{lccc}
\hline & $\begin{array}{c}\text { VAD after } \\
\text { transplant } \\
\text { (\% of all } \\
\text { transplants) }\end{array}$ & $\begin{array}{c}\text { Number } \\
\text { weaned } \\
(\%)\end{array}$ & $\begin{array}{c}\text { Discharge } \\
(\%)\end{array}$ \\
\hline Current study (2002) & $7(2.0)$ & $7(100)$ & $5(71)$ \\
Minev and colleagues (2001) & $15(2.1)$ & $7(47)$ & $3(20)$ \\
Arafa and colleagues $(2001)^{2}$ & $16(5.2)^{*}$ & $11(68)$ & $4(25)$ \\
Reiss and colleagues (2000) & $9(1.1)$ & $6(66)$ & $3(33)$ \\
Tenderich and colleagues (1998) & $25(2.9)$ & $5(20)$ & $6(24)$ \\
Barnard and colleagues (1995) & $6(1.9)$ & $2(33)$ & $2(33)$ \\
Frazier and colleagues (1990) & $3(1.2)$ & $3(100)$ & $1(33)$ \\
Radovancevic and colleagues & $4(1.5)^{*}$ & $4(100)$ & $2(50)$ \\
$\quad(1989)^{7}$ & & & \\
\hline
\end{tabular}

*These studies included intra-aortic balloon pump (IABP) placement.

measures that should be used before consideration of BVS5000 implantation. For severe rejection cases, cellular rejection was treated with high-dose methylprednisolone, increased doses of cyclosporine, and antilymphocyte antibody preparations; in addition, potential humoral rejection was treated with plasmapheresis, IVIG, and cyclophosphamide. For severe rejection, we again advocate percutaneous IABP and inotropic support as less invasive initial treatments. Nevertheless, multiple reports describe transplant patients who require complete mechanical ventricular support. Table 4 summarizes reports of cardiac transplant patients who were completely supported with mechanical ventricular assist devices.

In these previous reports, a variety of devices were used including ECMO, centrifugal vortex pumps (MedtronicBioMedicus, Eden Prairie, Minn), intra-aortic axial flow pumps, and Thoratec VADs. Again, primary graft failure and severe acute rejection with cardiogenic shock comprise the primary etiologies that necessitated mechanical support. Despite the encouraging results of two small, early series in the late 1980s, the results of recent reports generally have not encouraged the use of mechanical support in the heart transplant patient. ${ }^{1-7}$ Overall survival of these patients has been poor, with substantial morbidity (Table 4).

The positive results in the transplant patients in this study, relative to those seen in recent series, may be related to the early systematic application of VAD support, as well as to the type of VAD support. We have found the BVS5000 to be easy to implant, manage, and explant. Early systematic application of the BVS5000 has avoided secondary complications that accompany circulatory failure.

Additionally, although there are only 7 patients in our series, outcomes with the BVS5000 in cardiac transplant patients appear better relative to other patients we have supported with this device (Table 2). Notably, all the transplant patients could be weaned from the device and all experienced ventricular recovery. In contrast, among the nontransplant group, only $13 \%$ were weaned and only 1 patient in this group experienced long-term survival with the native heart. These results emphasize that the pathophysiology instigating cardiogenic shock and the potential for ventricular recovery are fundamentally different in these 2 groups. Results of the nontransplant patients demonstrate the improved outcomes with the BVS5000 were not an overall trend but may be limited to the transplanted hearts.

We advocate the BVS5000 because it can be rapidly installed in the transplanted heart, is easily managed, and can be removed without need for cardiopulmonary bypass. In contrast, IABP may not provide the complete ventricular support needed during these conditions and certainly does not provide significant right ventricular support, which is often important in the transplanted heart. Biomedicus pumps and ECMO circuits generally require continuous supervision by a perfusionist and may include risks for additional complications (for example, limb ischemia from percutaneous femoral cannulation). Finally, more permanent VADs often require ventricular cannulation, making explantation more difficult. Such long-term support is usually not necessary and, additionally, these long-term devices are limited by higher cost.

The high rate at which the BVS5000 could be weaned in this series emphasizes the recoverability of ventricular function in the transplanted heart during primary graft failure or severe acute rejection with cardiogenic shock. The transplanted hearts recovered better than hearts supported for other nontransplant conditions such as postcardiotomy shock or viral myocarditis. On the basis of these results, we advocate consideration of a systematic early application of the BVS5000 for cardiac transplant patients with either primary graft failure or severe acute rejection with cardiogenic shock. Ventricular function appears to recover in both of these conditions. This report does identify a higher incidence of renal insufficiency for the transplant group. This may result from the concomitant use of the immunosuppressant cyclosporine, which can cause renal insufficiency. Notably, none of the patients in the transplant group required permanent dialysis. Surprisingly, other complications such as sepsis were not increased in the transplant group. Finally, despite increased renal insufficiency, the BVS5000 successfully supported the failing donor heart, allowing long-term survival in a majority of transplanted patients.

\section{References}

1. Minev PV, El-Banayosy A, Minami K, Kortke H, Kizner L, Korfer R. Differential indications for mechanical circulatory support following heart transplantation. Intensive Care Med. 2001;27:1321-7.

2. Arafa O, Fiane AE, Svennevig JL, Gerian OR. Mechanical circulatory support of heart transplant patients. Transplant Proc. 2001; 33:1603-4. 
3. Reiss N, El-banayosy A, Mirow N, Minami K, Korfer R. Implantation of the Biomedicus centrifugal pump in post-transplant right heart failure. J Cardiovasc Surg. 2000;41:691-4.

4. Tenderich G, Koerner MM, Stuettgen B, Minami K, el Banayosy A, Arusoglu L. Mechanical circulatory support after orthotopic heart transplantation. Int J Artif Organs. 1998;21:414-6.

5. Barnard SP, Hasan A, Forty J, Hilton CJ, Dark JH. Mechanical ventricular assistance for the failing right ventricle after cardiac transplantation. Eur J Cardiothorac Surg. 1995;9:297-9.

6. Frazier OH, Macris MP, Wampler RK, Duncan JM, Sweeney MS, Fuqua JM. Treatment of cardiac allograft failure by use of an intraaortic axial flow pump. $J$ Heart Transplant. 1990;9:408-14.

7. Radovancevic B, Nakatani T, Frazier $\mathrm{OH}$, et al. Mechanical circulatory support for perioperative donor heart failure. ASAIO Transactions. 1989;35:539-41.

8. Dewey TM, Chen JM, Spanier TB, Oz MC. Alternative technique of right-sided outflow cannula insertion for right ventricular support. Ann Thorac Surg. 1998;66:1829-30.

9. Samuels LE, Holmes EC, Thomas MP, et al. Management of acute cardiac failure with mechanical assist: experience with the ABIOMED BVS 5000. Ann Thorac Surg. 2001;71:S67-72.

10. Dekkers RJ, Fitzgerald DJ, Couper GS. Five-year clinical experience with Abiomed BVS 5000 as a ventricular assist device for cardiac failure. Perfusion. 2001;16:13-8.

11. Sato M, Katayama Y, Higuchi S, Kosako Y, Ohtsubo S, Itoh T. Initial clinical experiences of the ABIOMED BVS SYSTEM 5000 in Japan. Jpn J Thorac Cardiovasc Surg. 1998;46:236-42.

12. Jett GK. ABIOMED BVS 5000: experience and potential advantages. Ann Thorac Surg. 1996;61:301-4.

13. Jahania MS, Mullett TW, Sanchez JA, Narayan P, Lasley RD, Mentzer RM. Acute allograft failure in thoracic organ transplantation. $J$ Card Surg. 2000;15:122-8.

\section{Availability of Journal back issues}

As a service to our subscribers, copies of back issues of The Journal of Thoracic and Cardiovascular Surgery for the preceding 5 years are maintained and are available for purchase from Mosby until inventory is depleted. Please write to Mosby, Subscription Customer Service, 6277 Sea Harbor Dr, Orlando, FL 32877, or call $800-654-2452$ or $407-345-4000$ for information on availability of particular issues and prices. 